\title{
Ccreative
commons
}

ISSN 1855-3966 (printed edn.), ISSN 1855-3974 (electronic edn.)

\author{
ARS MATHEMATICA CONTEMPORANEA 20 (2021) 129-142 \\ https://doi.org/10.26493/1855-3974.2227.e1a \\ (Also available at http://amc-journal.eu)
}

\section{The enclaveless competition game}

\author{
Michael A. Henning * \\ Department of Mathematics and Applied Mathematics, University of Johannesburg, \\ Auckland Park, 2006 South Africa \\ Douglas F. Rall \\ Professor Emeritus of Mathematics \\ Furman University, Greenville, SC, USA
}

Received 24 January 2020, accepted 11 November 2020, published online 19 August 2021

\begin{abstract}
For a subset $S$ of vertices in a graph $G$, a vertex $v \in S$ is an enclave of $S$ if $v$ and all of its neighbors are in $S$, where a neighbor of $v$ is a vertex adjacent to $v$. A set $S$ is enclaveless if it does not contain any enclaves. The enclaveless number $\Psi(G)$ of $G$ is the maximum cardinality of an enclaveless set in $G$. As first observed in 1997 by Slater, if $G$ is a graph with $n$ vertices, then $\gamma(G)+\Psi(G)=n$ where $\gamma(G)$ is the well-studied domination number of $G$. In this paper, we continue the study of the competition-enclaveless game introduced in 2001 by Philips and Slater and defined as follows. Two players take turns in constructing a maximal enclaveless set $S$, where one player, Maximizer, tries to maximize $|S|$ and one player, Minimizer, tries to minimize $|S|$. The competition-enclaveless game number $\Psi_{g}^{+}(G)$ of $G$ is the number of vertices played when Maximizer starts the game and both players play optimally. We study among other problems the conjecture that if $G$ is an isolate-free graph of order $n$, then $\Psi_{g}^{+}(G) \geq \frac{1}{2} n$. We prove this conjecture for regular graphs and for claw-free graphs.
\end{abstract}

Keywords: Competition-enclaveless game, domination game.

Math. Subj. Class. (2020): 05C65, 05C69

\section{Introduction}

In 2010 Brešar, Klavžar, and Rall [2] published the seminal paper on the domination game which belongs to the growing family of competitive optimization graph games. Domination games played on graphs are now very well studied in the literature. Indeed, the

* Research supported in part by the University of Johannesburg.

E-mail addresses: mahenning@uj.ac.za (Michael A. Henning), doug.rall@furman.edu (Douglas F. Rall)

()( This work is licensed under https://creativecommons.org/licenses/by/4.0/ 
subsequent rapid growth by the scientific community of research on domination games played on graphs resulted in several dozen papers to date on the domination-type games (see, for example, [3, 4, 10, 11, 12, 14, 17]). A recent book entitled "Domination games played on graphs" by Brešar, Henning, Klavžar, and Rall [1] presents the state of the art results to date, and shows that the area is rich for further research. In this paper, we continue the study of domination games played on graphs, and investigate in more depth the competition-enclaveless game birthed by Philips and Slater [15, 16].

A neighbor of a vertex $v$ in $G$ is a vertex that is adjacent to $v$. A vertex dominates itself and its neighbors. A dominating set of a graph $G$ is a set $S$ of vertices of $G$ such that every vertex in $G$ is dominated by a vertex in $S$. The domination number of $G$, denoted $\gamma(G)$, is the minimum cardinality of a dominating set in $G$, while the upper domination number of $G$, denoted $\Gamma(G)$, is the maximum cardinality of a minimal dominating set in $G$. A minimal dominating set of cardinality $\Gamma(G)$ we call a $\Gamma$-set of $G$.

The open neighborhood of a vertex $v$ in $G$ is the set of neighbors of $v$, denoted $N_{G}(v)$. Thus, $N_{G}(v)=\{u \in V \mid u v \in E(G)\}$. The closed neighborhood of $v$ is the set $N_{G}[v]=$ $\{v\} \cup N_{G}(v)$. If the graph $G$ is clear from context, we simply write $N(v)$ and $N[v]$ rather than $N_{G}(v)$ and $N_{G}[v]$, respectively.

As defined by Alan Goldman and introduced by Slater in [19], for a subset $S$ of vertices in a graph $G$, a vertex $v \in S$ is an enclave of $S$ if it and all of its neighbors are also in $S$; that is, if $N[v] \subseteq S$. A set $S$ is enclaveless if it does not contain any enclaves. We note that a set $S$ is a dominating set of a graph $G$ if and only if the set $V(G) \backslash S$ is enclaveless. The enclaveless number of $G$, denoted $\Psi(G)$, is the maximum cardinality of an enclaveless set in $G$, and the lower enclaveless number of $G$, denoted by $\psi(G)$, is the minimum cardinality of a maximal enclaveless set. The domination and enclaveless numbers of a graph $G$ are related by the following equations.

Observation 1.1. If $G$ is a graph of order $n$, then $\gamma(G)+\Psi(G)=n=\Gamma(G)+\psi(G)$.

The domination game on a graph $G$ consists of two players, Dominator and Staller, who take turns choosing a vertex from $G$. Each vertex chosen must dominate at least one vertex not dominated by the vertices previously chosen. Upon completion of the game, the set of chosen (played) vertices is a dominating set in $G$. The goal of Dominator is to end the game with a minimum number of vertices chosen, while Staller has the opposite goal and wishes to end the game with as many vertices chosen as possible.

The Dominator-start domination game and the Staller-start domination game is the domination game when Dominator and Staller, respectively, choose the first vertex. We refer to these simply as the D-game and S-game, respectively. The game domination number, $\gamma_{\mathrm{g}}(G)$, of $G$ is the number of moves in a D-game when both players play optimal strategies consistent with their goals. The Staller-start game domination number, $\gamma_{\mathrm{g}}^{\prime}(G)$, of $G$ is defined analogously for the S-game.

Philips and Slater $[15,16]$ introduced what they called the competition-enclaveless game. The game is played by two players, Maximizer and Minimizer, on some graph $G$. They take turns in constructing a maximal enclaveless set $S$ of $G$. That is, in each turn a player plays a vertex $v$ that is not in the set $S$ of the vertices already chosen and such that $S \cup\{v\}$ does not contain an enclave, until there is no such vertex. We call such a vertex a playable vertex. The goal of Maximizer is to make the final set $S$ as large as possible and for Minimizer to make the final set $S$ as small as possible.

The competition-enclaveless game number, or simply the enclaveless game number, 
$\Psi_{g}^{+}(G)$, of $G$ is the number of vertices chosen when Maximizer starts the game and both players play an optimal strategy according to the rules. The Minimizer-start competition-enclaveless game number, or simply the Minimizer-start enclaveless game number, $\Psi_{g}^{-}(G)$, of $G$ is the number of vertices chosen when Minimizer starts the game and both players play an optimal strategy according to the rules. The competition-enclaveless game, which has been studied for example in $[8,9,15,16,18]$, has not yet been explored in as much depth as the domination game. In this paper we continue the study of the competitionenclaveless game. Our main motivation for our study are the following conjectures that have yet to be settled, where an isolate-free graph is a graph that does not contain an isolated vertex.

Conjecture 1.2. If $G$ is an isolate-free graph of order $n$, then $\Psi_{g}^{+}(G) \geq \frac{1}{2} n$.

Conjecture 1.2 was first posed as a question by Peter Slater to the 2nd author on 8th May 2015, and subsequently posed as a conjecture in [9]. We refer to Conjecture 1.2 for general isolate-free graphs as the $\frac{\mathbf{1}}{\mathbf{2}}$-Enclaveless Game Conjecture. We also pose the following conjecture for the Minimizer-start enclaveless game, where $\delta(G)$ denotes the minimum degree of the graph $G$.

Conjecture 1.3. If $G$ is a graph of order $n$ with $\delta(G) \geq 2$, then $\Psi_{g}^{-}(G) \geq \frac{1}{2} n$.

We proceed as follows. By Observation 1.1, if the domination number of a graph is known, then we immediately know the enclaveless number, and vice versa. In contrast, we show in Section 2 that the game domination number and the enclaveless game number are very different and are not related in the same way that the domination and enclaveless numbers are related. Indeed, knowledge of the game domination number gives no information of the enclaveless game number, and vice versa. We show that the domination game and the enclaveless game are intrinsically different. In Section 3, we present fundamental bounds on the enclaveless game number and the Minimizer-start enclaveless game number. In Sections 4 and 5, we show that the $\frac{1}{2}$-Enclaveless Game Conjecture holds for regular graphs and claw-free graphs, respectively. We use the standard notation $[k]=\{1, \ldots, k\}$.

\section{Game domination versus enclaveless game}

Although the domination and enclaveless numbers of a graph $G$ of order $n$ are related by the equation $\gamma(G)+\Psi(G)=n$ (see Observation 1.1), as remarked in [9] the competitionenclaveless game is very different from the domination game. To illustrate this, we present two simple examples showing that the sum $\gamma_{\mathrm{g}}(G)+\Psi_{g}^{+}(G)$ on the class of graphs of a fixed order can differ greatly even when restricted to trees.

For the first example, for $k \geq 3$, let $G$ be a tree with exactly two non-leaf vertices both of which have $k$ leaf neighbors, that is, $G$ is a double star $S(k, k)$. In this case, $\Psi_{g}^{+}(G)=\Psi_{g}^{-}(G)=k+1$ and $\gamma_{\mathrm{g}}(G)=3$ and $\gamma_{\mathrm{g}}^{\prime}(G)=4$. Thus if $G$ is a double star of order $n$, then $\gamma_{\mathrm{g}}(G)+\Psi_{g}^{+}(G)=\frac{1}{2} n+3$.

For the second example, we consider the class of paths; $P_{n}$ denotes the path on $n$ vertices. For $n \geq 1$, Košmrlj [14] showed that $\gamma_{\mathrm{g}}^{\prime}\left(P_{n}\right)=\left\lceil\frac{n}{2}\right\rceil$ and that $\gamma_{\mathrm{g}}\left(P_{n}\right)=\left\lceil\frac{n}{2}\right\rceil-1$ if $n \equiv 3(\bmod 4)$ and $\gamma_{\mathrm{g}}\left(P_{n}\right)=\left\lceil\frac{n}{2}\right\rceil$, otherwise. For $n \geq 2$, Phillips and Slater [16] showed that $\Psi_{g}^{+}\left(P_{n}\right)=\left\lfloor\frac{3 n+1}{5}\right\rfloor$ and $\Psi_{g}^{-}\left(P_{n}\right)=\left\lfloor\frac{3 n}{5}\right\rfloor$. Thus if $G$ is a path $P_{n}$, then $\gamma_{\mathrm{g}}(G)+\Psi_{g}^{+}(G) \approx n+\frac{1}{10} n$. 
The most important general fact in the domination game is the so-called Continuation Principle, which provides a much-used monotonicity property of the game domination number and allows us to assume that each optimal move of Dominator (and of Staller) is taken from a restricted subset of the unchosen vertices. Due to its importance in the domination game, we recall this well-studied Continuation Principle. A partially dominated graph is a graph together with a declaration that some vertices are already dominated and need not be dominated, but can be played, in the rest of the game. Given a graph $G$ and a subset $S$ of vertices of $G$, we denote by $G \mid S$ the partially dominated graph with $S$ as the set of declared vertices already dominated. We use $\gamma_{\mathrm{g}}(G \mid S)$ (resp. $\gamma_{\mathrm{g}}^{\prime}(G \mid S)$ ) to denote the number of moves remaining in the game on $G \mid S$ under optimal play when Dominator (resp. Staller) has the next move. We are now in a position to state the Continuation Principle presented by Kinnersley, West, and Zamani in [12, Lemma 2.1].

Lemma 2.1 (Continuation Principle). If $G$ is a graph and $A, B \subseteq V(G)$ with $B \subseteq A$, then $\gamma_{\mathrm{g}}(G \mid A) \leq \gamma_{\mathrm{g}}(G \mid B)$ and $\gamma_{\mathrm{g}}^{\prime}(G \mid A) \leq \gamma_{\mathrm{g}}^{\prime}(G \mid B)$.

The Continuation Principle is one of the most important proof techniques to obtain results on the domination game and its variants. It yields, for example, the following fundamental monotonicity property of the domination game; see [2, Theorem 6] and [12, Corollary 4.1].

Theorem 2.2. The Dominator-start game domination number and the Staller-start game domination number can differ by at most 1 , that is, for any graph $G$, we have

$$
\left|\gamma_{\mathrm{g}}(G)-\gamma_{\mathrm{g}}^{\prime}(G)\right| \leq 1
$$

The most significant difference between the competition-enclaveless game and the domination game is that the Continuation Principle holds for the domination game but does not hold for the competition-enclaveless game. If the Continuation Principle were to hold for the competition-enclaveless game, then this would imply that the Maximizer-start enclaveless game number and the Minimizer-start enclaveless game number can differ by at most 1 . However, this is not the case, and these two game numbers can differ significantly. For example, if $n \geq 1$ and $G$ is a star $K_{1, n}$, then $\Psi_{g}^{+}(G)=n$ while $\Psi_{g}^{-}(G)=1$. Thus, the numbers $\Psi_{g}^{+}(G)$ and $\Psi_{g}^{-}(G)$ can vary greatly.

Without the powerful proof method of the Continuation Principle at our disposal, the competition-enclaveless game is raised to a greater level of difficulty than other domination games played on graphs. Indeed, this suggests that there may exist a graph $G$ (or infinite classes of graphs) for which $\Psi_{g}^{-}(G)>\Psi_{g}^{+}(G)$ is possible, and such that the difference $\Psi_{g}^{-}(G)-\Psi_{g}^{+}(G)$ can possibly be made arbitrarily large. However, we are unable at this time to construct such examples, if they exist. Moreover, we are also unable to prove at this time that $\Psi_{g}^{-}(G) \leq \Psi_{g}^{+}(G)$ is always true.

Another significant difference between the domination game and the competition-enclaveless game is that upon completion of the domination game, the set of played vertices is a dominating set although not necessarily a minimal dominating set, while upon completion of the competition-enclaveless game, the set of played vertices is always a maximal enclaveless set. Thus, the enclaveless game numbers of a graph $G$ are always squeezed between the lower enclaveless number $\psi(G)$ of $G$ and the enclaveless number $\Psi(G)$ of $G$. We state this formally as follows. 
Observation 2.3. If $G$ is a graph, then

$$
\psi(G) \leq \Psi_{g}^{-}(G) \leq \Psi(G) \quad \text { and } \quad \psi(G) \leq \Psi_{g}^{+}(G) \leq \Psi(G) .
$$

A graph $G$ is well-dominated if all the minimal dominating sets of $G$ have the same cardinality. Examples of well-dominated graphs include, for example, the complete graph $K_{n}, C_{7}, P_{4}$, the corona of any graph, and the graph formed from two vertex disjoint cycles of order 5 joined by a single edge. Finbow, Hartnell and Nowakowski [7] characterized the well-dominated graphs having no 3-cycle nor 4-cycle. As observed earlier, upon completion of the enclaveless game, the set of played vertices is always a maximal enclaveless set. Hence, any sequence of legal moves by Maximizer and Minimizer (regardless of strategy) in the enclaveless game played in a well-dominated graph of order $n$ will always lead to the game ending in $n-\gamma(G)$ moves. Thus as a consequence of Observation 2.3, we have the following interesting connection between the enclaveless game and the class of well-dominated graphs.

Observation 2.4. If $G$ is a well-dominated graph of order $n$, then

$$
\Psi_{g}^{-}(G)=\Psi_{g}^{+}(G)=n-\gamma(G) .
$$

It is well-known that if $G$ is an isolate-free graph of order $n$, then $\gamma(G) \leq \frac{1}{2} n$, implying by Observation 1.1 that $\Psi(G)=n-\gamma(G) \geq \frac{1}{2} n$. Hence one might think that $\gamma_{g}(G) \leq$ $\Psi_{g}^{+}(G)$ for such a graph $G$ with no isolated vertex. We now provide an infinite class of graphs to show that the ratio $\gamma_{g} / \Psi_{g}^{+}$of these two graphical invariants can be strictly larger than, and bounded away from, 1 . The corona $\operatorname{cor}(G)$ of a graph $G$, also denoted $G \circ K_{1}$ in the literature, is the graph obtained from $G$ by adding for each vertex $v$ of $G$ a new vertex $v^{\prime}$ and the edge $v v^{\prime}$ (and so, the vertex $v^{\prime}$ has degree 1 in $\operatorname{cor}(G)$ ). The edge $v v^{\prime}$ is called a pendant edge. We shall need the following 2014 result due to Košmrlj [13].

Theorem 2.5 ([13, Theorem 4.1]). For $k \geq 1$, if $G=\operatorname{cor}\left(P_{k}\right)$, then $\gamma_{\mathrm{g}}(G)=k+\left\lceil\frac{k-7}{10}\right\rceil$.

Let $\mathcal{G}$ be the (infinite) family of coronas of paths $P_{k}$ where $k \geq 8$ and $k \bmod 10 \in$ $\{8,9\}$, that is,

$$
\mathcal{G}=\left\{\operatorname{cor}\left(P_{k}\right): k \bmod 10 \in\{8,9\}\right\} .
$$

As a consequence of Theorem 2.5, we have the following result.

Theorem 2.6. For every graph $G \in \mathcal{G}$, we have

$$
\frac{\gamma_{g}(G)}{\Psi_{g}^{+}(G)}>\frac{11}{10} \text {. }
$$

Proof. Let $G \in \mathcal{G}$, and so $G=\operatorname{cor}\left(P_{k}\right)$ for some positive integer $k$ where $k \bmod 10 \in$ $\{8,9\}$. Every minimal dominating set of $G$ has cardinality $k$, which implies by Observation 1.1 that every maximal enclaveless set of $G$ also has cardinality $k$; that is, $\psi(G)=$ $\Psi(G)=k$ where we recall that $\psi(G)$ denotes the cardinality of the smallest maximal enclaveless set in $G$ and $\Psi(G)$ is the cardinality of a largest enclaveless set in $G$. Hence by Observation $2.3, \Psi_{g}^{+}(G)=k$. Consequently, by Theorem 2.5 and since $k \bmod 10 \in\{8,9\}$ we have

$$
\frac{\gamma_{g}(G)}{\Psi_{g}^{+}(G)}=\frac{k+\left\lceil\frac{k-7}{10}\right\rceil}{k}>\frac{11}{10} .
$$

Hence, by Theorem 2.6, the difference $\gamma_{g}(G)-\Psi_{g}^{+}(G)$ can be made arbitrarily large for an infinite family of graphs. 


\section{Fundamental bounds}

In this section, we establish some fundamental bounds on the (Maximizer-start) enclaveless game number and the Minimizer-start enclaveless game number. We establish next a lower and upper bound on the enclaveless number of a graph in terms of the maximum degree and order of the graph.

Proposition 3.1. If $G$ is an isolate-free graph of order $n$ with maximum degree $\Delta(G)=\Delta$, then

$$
\left(\frac{1}{\Delta+1}\right) n \leq \psi(G) \leq \Psi(G) \leq\left(\frac{\Delta}{\Delta+1}\right) n
$$

Proof. If $G$ is any graph of order $n$ and maximum degree $\Delta$, then $\gamma(G) \geq \frac{n}{\Delta+1}$. Hence, by Observation 1.1,

$$
\Psi(G)=n-\gamma(G) \leq n-\frac{n}{\Delta+1}=\left(\frac{\Delta}{\Delta+1}\right) n .
$$

On the other hand, let $D$ be a minimal dominating set of maximum cardinality, and so $|D|=\Gamma(G)$. Let $\bar{D}=V(G) \backslash D$, and so $|\bar{D}|=n-|D|$. Let $\ell$ be the number of edges between $D$ and $\bar{D}$. Since $G$ is an isolate-free graph and $D$ is a minimal dominating set, every vertex in $D$ has at least one neighbor in $\bar{D}$, and so $\ell \geq|D|$. Since $G$ has maximum degree $\Delta$, every vertex in $\bar{D}$ has at most $\Delta$ neighbors in $D$, and so $\ell \leq \Delta \cdot|\bar{D}|=$ $\Delta(n-|D|)$. Hence, $|D| \leq \Delta(n-|D|)$, implying that $\Gamma(G)=|D| \leq \Delta n /(\Delta+1)$. Thus by Observation 1.1,

$$
\psi(G)=n-\Gamma(G) \geq n-\left(\frac{\Delta}{\Delta+1}\right) n=\left(\frac{1}{\Delta+1}\right) n .
$$

This completes the proof of Proposition 3.1.

By Observation 2.3, the set of played vertices in either the Maximizer-start enclaveless game or the Minimizer-start enclaveless game is a maximal enclaveless set of $G$. Thus as an immediate consequence of Proposition 3.1, we have the following result.

Corollary 3.2. If $G$ is an isolate-free graph of order $n$ with maximum degree $\Delta(G)=\Delta$, then

$$
\left(\frac{1}{\Delta+1}\right) n \leq \Psi_{g}^{-}(G) \leq\left(\frac{\Delta}{\Delta+1}\right) n \quad \text { and } \quad\left(\frac{1}{\Delta+1}\right) n \leq \Psi_{g}^{+}(G) \leq\left(\frac{\Delta}{\Delta+1}\right) n \text {. }
$$

We show that the upper bounds in Corollary 3.2 are realized for infinitely many connected graphs.

Proposition 3.3. There exist infinitely many positive integers $n$ along with a connected graph $G$ of order $n$ satisfying

$$
\Psi_{g}^{-}(G)=\Psi_{g}^{+}(G)=\left(\frac{\Delta(G)}{\Delta(G)+1}\right) n .
$$

Proof. Let $r$ be an integer such that $r \geq 4$ and let $m$ be any positive integer. For each $i \in[m]$, let $H_{i}$ be a graph obtained from a complete graph of order $r+1$ by removing 
the edge $x_{i} y_{i}$ for two distinguished vertices $x_{i}$ and $y_{i}$. The graph $F_{m}$ is obtained from the disjoint union of $H_{1}, \ldots, H_{m}$ by adding the edges $y_{i} x_{i+1}$ for each $i \in[m]$ where the subscripts are computed modulo $m$. The vertices $x_{i}$ and $y_{i}$ are called connectors in $F_{m}$, and each of the $r-1$ vertices in the set $V\left(H_{i}\right) \backslash\left\{x_{i}, y_{i}\right\}$ is called a hidden vertex of $H_{i}$. Note that $F_{m}$ is $r$-regular and has order $n=m(r+1)$.

We first show that $\Psi_{g}^{-}\left(F_{m}\right)=\left(\frac{r}{r+1}\right) n$. Suppose the Minimizer-start enclaveless game is played on $F_{m}$. We provide a strategy for Maximizer that forces exactly $r m$ vertices to be played. Maximizer's strategy is to make sure that all the connector vertices in the graph are played. If he can accomplish this, then exactly $\mathrm{rm}$ vertices will be played when the game ends because of the structure of $F_{m}$. Suppose that at some point in the game Minimizer plays a vertex from some $H_{j}$. If one of the connector vertices, say $x_{j}$, is playable, then Maximizer responds by playing $x_{j}$. If both connector vertices have already been played and some hidden vertex, say $w$, in $H_{j}$ is playable, then Maximizer plays $w$. If no vertex of $H_{j}$ is playable, then Maximizer plays a connector vertex from $H_{i}$ for some $i \neq j$ if one is playable and otherwise plays any playable vertex. Since $H_{k}$ contains at least 3 hidden vertices for each $k \in[m]$, it follows that Maximizer can guarantee that all the connector vertices are played by following this strategy. This implies that for each $i \in[m]$, exactly one hidden vertex of $H_{i}$ is not played during the course of the game. That is, the set of played vertices has cardinality

$$
r m=\left(\frac{r}{r+1}\right) m(r+1)=\left(\frac{\Delta\left(F_{m}\right)}{\Delta\left(F_{m}\right)+1}\right) n,
$$

where we recall that $\Delta\left(F_{m}\right)=r$. Thus,

$$
\Psi_{g}^{-}\left(F_{m}\right) \geq\left(\frac{\Delta\left(F_{m}\right)}{\Delta\left(F_{m}\right)+1}\right) n .
$$

By Corollary 3.2,

$$
\Psi_{g}^{-}\left(\left(F_{m}\right)\right) \leq\left(\frac{\Delta\left(F_{m}\right)}{\Delta\left(F_{m}\right)+1}\right) n .
$$

Consequently, $\Psi_{g}^{-}\left(F_{m}\right)=\left(\frac{\Delta\left(F_{m}\right)}{\Delta\left(F_{m}\right)+1}\right) n$.

If the Maximizer-start enclaveless game is played on $F_{m}$, then the same strategy as above for Maximizer forces $r m$ vertices to be played (even with the relaxed condition that $r$ be an integer larger than 2). Thus as before, $\Psi_{g}^{+}\left(F_{m}\right)=\left(\frac{\Delta\left(F_{m}\right)}{\Delta\left(F_{m}\right)+1}\right) n$.

The lower bound in Corollary 3.2 on $\Psi_{g}^{-}(G)$ is achieved, for example, by taking $G=$ $K_{1, \Delta}$ for any given $\Delta \geq 1$ in which case $\Psi_{g}^{-}(G)=1=\left(\frac{1}{\Delta+1}\right) n$ where $n=n(G)=$ $\Delta+1$.

The lower bound in Corollary 3.2 on $\Psi_{g}^{+}(G)$ is trivially achieved when $\Delta=1$, in which case $G$ is the disjoint union of copies of $K_{2}$. However, as remarked in the introductory section, the main open problem in the competition-enclaveless game is the $\frac{1}{2}$-Enclaveless Game Conjecture (stated formally in Conjecture 1.2) that claims that if $G$ is an isolate-free graph of order $n$, then $\Psi_{g}^{+}(G) \geq \frac{1}{2} n$. If the conjecture is true, then, from our earlier examples such as the double star, the bound is achieved for isolate-free graphs with arbitrarily large maximum degree $\Delta$. 


\section{Regular graphs}

In this section, we show that $\frac{1}{2}$-Enclaveless Game Conjecture (see Conjecture 1.2) holds for the class of regular graphs, as does Conjecture 1.3 for the Minimizer-start enclaveless game. For a set $S \subset V(G)$ of vertices in a graph $G$ and a vertex $v \in S$, we define the $S$-external private neighborhood of a vertex $v$, abbreviated $\operatorname{epn}_{G}(v, S)$, as the set of all vertices outside $S$ that are adjacent to $v$ but to no other vertex of $S$; that is,

$$
\operatorname{epn}_{G}(v, S)=\left\{w \in V(G) \backslash S: N_{G}(w) \cap S=\{v\}\right\} .
$$

As remarked in the introduction, if the graph $G$ is clear from the context, we omit the subscript $G$ in the above definitions. We define an $S$-external private neighbor of $v$ to be a vertex in $\operatorname{epn}(v, S)$.

We establish next a $\frac{1}{2}$-lower bound on $\Psi_{g}^{+}(G)$ and $\Psi_{g}^{-}(G)$ by forbidding induced stars of a certain size. We remark that the proof of the following result uses similar counting techniques to those employed by Southey and Henning in [20].

Proposition 4.1. If $G$ is a graph with order $n$, minimum degree $\delta$ and with no induced $K_{1, \delta+1}$, then $\psi(G) \geq \frac{1}{2} n$.

Proof. Let $D$ be an arbitrary minimal dominating set of $G$. Denote by $D_{1}$ the set of vertices in $D$ that have a $D$-external private neighbor. That is, $D_{1}=\{x \in D: \operatorname{epn}(x, D) \neq \emptyset\}$. In addition, let $D_{2}=D \backslash D_{1}$. Since $D$ is a minimal dominating set, the set $D_{2}$ consists of those vertices in $D$ that are isolated in the subgraph $G[D]$ of $G$ induced by $D$. Let

$$
C_{1}=\bigcup_{x \in D_{1}} \operatorname{epn}(x, D) \quad \text { and } \quad C_{2}=V(G) \backslash\left(D \cup C_{1}\right) .
$$

We note that by definition, there are no edges in $G$ joining a vertex in $D_{2}$ and a vertex in $C_{1}$. That is, each vertex in $D_{2}$ has at least $\delta$ neighbors in $C_{2}$. Since the set $D_{2}$ is independent and $G$ has no induced $K_{1, \delta+1}$, each vertex of $C_{2}$ has at most $\delta$ neighbors in $D_{2}$. Denote by $\ell$ the number of edges of the form $u v$ where $u \in D_{2}$ and $v \in C_{2}$. It now follows that $\delta\left|D_{2}\right| \leq \ell \leq \delta\left|C_{2}\right|$, that is, $\left|D_{2}\right| \leq\left|C_{2}\right|$. Now,

$$
|D|=\left|D_{1}\right|+\left|D_{2}\right| \leq\left|C_{1}\right|+\left|C_{2}\right|=n-|D|,
$$

which shows that $\Gamma(G) \leq \frac{1}{2} n$. Hence by Observation 1.1 , we have $\psi(G) \geq \frac{1}{2} n$.

Observation 2.3 and Proposition 4.1 now yield the following result.

Corollary 4.2. If $G$ is a graph with order $n$, minimum degree $\delta$ and with no induced $K_{1, \delta+1}$, then $\Psi_{g}^{+}(G) \geq \frac{1}{2} n$ and $\Psi_{g}^{-}(G) \geq \frac{1}{2} n$.

As a special case of Corollary 4.2, we have the desired $\frac{1}{2}$-lower bound on $\Psi_{g}^{+}(G)$ and $\Psi_{g}^{-}(G)$ for regular graphs $G$ without isolated vertices.

Corollary 4.3. If $G$ is a regular graph of order $n$ without isolated vertices, then $\Psi_{g}^{+}(G) \geq$ $\frac{1}{2} n$ and $\Psi_{g}^{-}(G) \geq \frac{1}{2} n$.

We remark that if $G$ is a graph of order $n$ that is a disjoint union of copies of $K_{2}$, then $\Psi_{g}^{+}(G)=\frac{1}{2} n$ and $\Psi_{g}^{-}(G)=\frac{1}{2} n$. The same conclusion holds if $G$ is a disjoint union of copies of $C_{4}$. Hence, for $k \in\{1,2\}$ there are $k$-regular graphs $G$ that achieve equality in the lower bound in Corollary 4.3. However, it remains an open problem to characterize the graphs achieving equality in Corollary 4.3 for each value of $k \geq 3$. 


\section{Claw-free graphs}

A graph is claw-free if it does not contain the star $K_{1,3}$ as an induced subgraph. In this section, we show that $\frac{1}{2}$-Enclaveless Game Conjecture (see Conjecture 1.2) holds for the class of claw-free graphs with no isolated vertex, as does Conjecture 1.3 for the Minimizerstart enclaveless game. For this purpose, we recall the definition of an irredundant set. For a set $S$ of vertices in a graph $G$ and a vertex $v \in S$, the $S$-private neighborhood of $v$ is the set

$$
\operatorname{pn}_{G}[v, S]=\{w \in V: N[w] \cap S=\{v\}\} .
$$

If the graph $G$ is clear from context, we simply write $\operatorname{pn}[v, S]$ rather than $\operatorname{pn}_{G}[v, S]$. We note that $\operatorname{epn}(v, S) \subseteq \operatorname{pn}[v, S] \subseteq \operatorname{epn}(v, S) \cup\{v\}$ and $v \in \operatorname{pn}[v, S]$ if and only if $v$ is isolated in $G[S]$. A vertex in the set $\operatorname{pn}[v, S]$ is called an $S$-private neighbor of $v$. The set $S$ is an irredundant set if every vertex of $S$ has an $S$-private neighbor. The upper irredundance number $\operatorname{IR}(G)$ is the maximum cardinality of an irredundant set in $G$.

The independence number $\alpha(G)$ of $G$ is the maximal cardinality of an independent set of vertices in $G$. An independent set of vertices of $G$ of cardinality $\alpha(G)$ is called an $\alpha$-set of $G$. Every maximum independent set in a graph is a minimal dominating set, and every minimal dominating set is an irredundant set. Hence we have the following inequality chain.

Observation 5.1 ([5]). For every graph $G$, we have $\alpha(G) \leq \Gamma(G) \leq \operatorname{IR}(G)$.

The inequality chain in Observation 5.1 is part of the canonical domination chain which was first observed by Cockayne, Hedetniemi, and Miller [5] in 1978.

In 2004, Favaron [6] established the following upper bound on the upper irredundance number of a claw-free graph.

Theorem 5.2 ([6]). If $G$ is a connected, claw-free graph of order $n$, then $\operatorname{IR}(G) \leq$ $\frac{1}{2}(n+1)$. Moreover, if $\operatorname{IR}(G)=\frac{1}{2}(n+1)$, then $\alpha(G)=\Gamma(G)=\operatorname{IR}(G)$.

In addition, she proved the following stronger upper bound for the upper irredundance number of claw-free graphs with minimum degree at least 2 .

Corollary 5.3 ([6]). If $G$ is a connected, claw-free graph of order $n$ and minimum degree at least 2 , then $\operatorname{IR}(G) \leq \frac{1}{2} n$.

Suppose that $G$ is a claw-free graph of order $n$ and minimum degree $\delta \geq 2$. By Corollary 5.3, $\operatorname{IR}(G) \leq \frac{1}{2} n$, and thus by Observations 1.1 and 5.1, we have

$$
\psi(G)=n-\Gamma(G) \geq n-\operatorname{IR}(G) \geq n-\frac{1}{2} n=\frac{1}{2} n .
$$

By Observation 2.3, we therefore have the following result.

Theorem 5.4. If $G$ is a claw-free graph of order $n$ and $\delta(G) \geq 2$, then

$$
\Psi_{g}^{+}(G) \geq \frac{1}{2} n \quad \text { and } \quad \Psi_{g}^{-}(G) \geq \frac{1}{2} n .
$$

By Theorem 5.4, we note that Conjecture 1.3 holds for connected claw-free graphs. In order to prove that Conjecture 1.2 holds for connected claw-free graphs, we need the 
characterization due to Favaron [6] of the graphs achieving equality in the bound of Theorem 5.2. For this purpose, we recall that a vertex $v$ of a graph $G$ is a simplicial vertex if its open neighborhood $N(v)$ induces a complete subgraph of $G$. A clique of a graph $G$ is a maximal complete subgraph of $G$. The clique graph of $G$ has the set of cliques of $G$ as its vertex set, and two vertices in the clique graph are adjacent if and only if they intersect as cliques of $G$. A non-trivial tree is a tree of order at least 2 .

Favaron [6] defined the family $\mathcal{F}$ of claw-free graphs $G$ as follows. Let $T_{1}, \ldots, T_{q}$ be $q \geq 1$ non-trivial trees. Let $L_{i}$ be the line graph of the corona $\operatorname{cor}\left(T_{i}\right)$ of the tree $T_{i}$ for $i \in[q]$. If $q=1$, let $G=L_{1}$. If $q \geq 2$, let $G$ be the graph constructed from the line graphs $L_{1}, L_{2}, \ldots, L_{q}$ by choosing $q-1$ pairs $\left\{x_{i j}, x_{j i}\right\}$ such that the following holds.

- $x_{i j}$ and $x_{j i}$ are simplicial vertices of $L_{i}$ and $L_{j}$, respectively, where $i \neq j$.

- The $2(q-1)$ vertices from the $q-1$ pairs $\left\{x_{i j}, x_{j i}\right\}$ are all distinct vertices.

- Contracting each pair of vertices $x_{i j}$ and $x_{j i}$ into one common vertex $c_{i j}$ results in a graph whose clique graph is a tree.

To illustrate the above construction of a graph $G$ in the family $\mathcal{F}$ consider, for example, such a construction when $q=3$ and the trees $T_{1}, T_{2}, T_{3}$ are given in Figure 1 .



Figure 1: An illustration of the construction of a graph $G$ in the family $\mathcal{F}$.

We note that if $G$ is an arbitrary graph of order $n$ in the family $\mathcal{F}$, then $n \geq 3$ is odd and the vertex set of $G$ can be partitioned into two sets $A$ and $B$ such that the following holds.

- $|A|=\frac{1}{2}(n-1)$ and $|B|=\frac{1}{2}(n+1)$.

- The set $B$ is an independent set. 
- Each vertex in $A$ has exactly two neighbors in $B$.

We refer to the partition $(A, B)$ as the partition associated with $G$. For the graph $G \in \mathcal{F}$ illustrated in Figure 1, the set $A$ consists of the darkened vertices and the set $B$ consists of the white vertices.

We are now in a position to state the characterization due to Favaron [6] of the graphs achieving equality in the bound of Theorem 5.2.

Theorem 5.5 ([6]). If $G$ is a connected, claw-free graph of order $n \geq 3$, then $\operatorname{IR}(G) \leq$ $\frac{1}{2}(n+1)$, with equality if and only if $G \in \mathcal{F}$.

We prove next the following property of graphs in the family $\mathcal{F}$.

Lemma 5.6. If $G \in \mathcal{F}$ and $(A, B)$ is the partition associated with $G$, then the set $B$ is the unique IR-set of $G$.

Proof. We proceed by induction on the order $n \geq 3$ of $G \in \mathcal{F}$. If $n=3$, then $G=P_{3}$. In this case, the set $B$ consists of the two leaves of $G$, and the desired result is immediate. This establishes the base case. Suppose that $n \geq 5$ and that the result holds for all graphs $G^{\prime} \in \mathcal{F}$ of order $n^{\prime}$, where $3 \leq n^{\prime}<n$. Let $Q$ be an IR-set of $G$.

By construction of the graph $G$, the set $B$ contains at least two vertices of degree 1 in $G$. Let $v$ be an arbitrary vertex in $B$ of degree 1 in $G$, and let $u$ be its neighbor. We note that $u \in A$. Let $G^{\prime}=G-\{u, v\}$ and let $G^{\prime}$ have order $n^{\prime}$, and so $n^{\prime}=n-2$. Let $A^{\prime}=A \backslash\{u\}$ and $B^{\prime}=B \backslash\{v\}$. By construction of the graph $G$ and our choice of the vertex $v$, we note that $G^{\prime} \in \mathcal{F}$ and that $\left(A^{\prime}, B^{\prime}\right)$ is the partition associated with $G^{\prime}$. Applying the inductive hypothesis to $G^{\prime}$, the set $B^{\prime}$ is the unique IR-set of $G^{\prime}$. Let $w$ be the second neighbor of $u$ in $G$ that belongs to the set $B$, and so $N(u) \cap B=\{v, w\}$. By the structure of the graph $G \in \mathcal{F}$, we note that $N[w] \subset N[u]$ and that the subgraph of $G$ induced by $N[w]$ is a clique.

Suppose, to the contrary, that $Q \neq B$. Let $Q^{\prime}$ be the restriction of $Q$ to the graph $G^{\prime}$, and so $Q^{\prime}=Q \cap V\left(G^{\prime}\right)$. Suppose that $u \in Q$. Since $Q$ is an irredundant set, this implies that $v \notin Q$. If $w \in Q$, then $\operatorname{pn}[w, Q]=\emptyset$, contradicting the fact that $Q$ is an irredundant set. Hence, $w \notin Q$, and so $Q^{\prime} \neq B^{\prime}$. By the inductive hypothesis, the set $Q^{\prime}$ is therefore not an IR-set of $G^{\prime}$, and so $\left|Q^{\prime}\right|<\operatorname{IR}\left(G^{\prime}\right)$. Thus, $\operatorname{IR}(G)=|Q|=\left|Q^{\prime}\right|+1 \leq$ $\left(\operatorname{IR}\left(G^{\prime}\right)-1\right)+1=\frac{1}{2}\left(n^{\prime}+1\right)=\frac{1}{2}(n-1)<\operatorname{IR}(G)$, a contradiction. Hence, $u \notin Q$. In this case, $\operatorname{IR}(G)=|Q| \leq\left|Q^{\prime}\right|+1 \leq \operatorname{IR}\left(G^{\prime}\right)+1=\frac{1}{2}\left(n^{\prime}+1\right)+1=\frac{1}{2}(n+1)=\operatorname{IR}(G)$. Hence, we must have equality throughout this inequality chain. This implies that $v \in Q$ and $\left|Q^{\prime}\right|=\operatorname{IR}\left(G^{\prime}\right)$. By the inductive hypothesis, we therefore have $Q^{\prime}=B^{\prime}$. Hence, $Q=Q^{\prime} \cup\{v\}=B^{\prime} \cup\{v\}=B$. Thus, the set $B$ is the unique IR-set of $G$.

Corollary 5.7. If $G \in \mathcal{F}$ and $(A, B)$ is the partition associated with $G$, then the set $B$ is the unique $\alpha$-set of $G$ and the unique $\Gamma$-set of $G$.

Proof. By Theorem 5.2, $\alpha(G)=\Gamma(G)=\operatorname{IR}(G)=\frac{1}{2}(n+1)$. By Lemma 5.6, the set $B$ is the unique IR-set of $G$. Since every $\alpha$-set of $G$ is an IR-set of $G$ and $\alpha(G)=\operatorname{IR}(G)$, this implies that $B$ is the unique $\alpha$-set of $G$. Since every $\Gamma$-set of $G$ is an IR-set of $G$ and $\Gamma(G)=\operatorname{IR}(G)$, this implies that $B$ is the unique $\Gamma$-set of $G$.

We show next that Conjecture 1.2 holds for connected claw-free graphs.

Theorem 5.8. If $G$ is a connected, claw-free graph of order $n \geq 2$, then the following holds. 
(a) $\Psi_{g}^{+}(G) \geq \frac{1}{2} n$.

(b) If $G \neq P_{3}$, then $\Psi_{g}^{-}(G) \geq \frac{1}{2} n$.

Proof. Let $G$ be a connected, claw-free graph of order $n \geq 2$. If $\operatorname{IR}(G) \leq \frac{1}{2} n$, then

$$
\min \left\{\Psi_{g}^{+}(G), \Psi_{g}^{-}(G)\right\} \geq \psi(G) \geq n-\operatorname{IR}(G) \geq \frac{1}{2} n
$$

Therefore, by Theorem 5.5, we can assume that $\operatorname{IR}(G)=\frac{1}{2}(n+1)$ and $G \in \mathcal{F}$. Let $(A, B)$ be the partition associated with $G$. We show in this case we have $\min \left\{\Psi_{g}^{+}(G), \Psi_{g}^{-}(G)\right\} \geq$ $\psi(G)+1$.

By Observation 1.1, $\Gamma(G)+\psi(G)=n$. Moreover, the complement of every $\Gamma$-set of $G$ is a maximal enclaveless set, and the complement of every $\psi$-set of $G$ is a minimal dominating set. By Corollary 5.7, the set $B$ is the unique $\Gamma$-set of $G$. These observations imply that the complement of the set $B$, namely the set $A$, is the unique $\psi$-set of $G$. Thus every maximal enclaveless set of $G$ of cardinality $\psi(G)$ is precisely the set $A$.

In the Maximizer-start enclaveless game played on $G$, Maximizer plays as his first move any vertex from the set $B$. In the Minimizer-start enclaveless game played on $G$, by supposition we have $G \neq P_{3}$, implying that there are at least two vertices in the set $B$ at distance at least 3 apart in $G$. Thus, whatever the first move is played by Minimizer, Maximizer can always respond by playing as his first move a vertex chosen from the set $B$. Hence, no matter who starts the game, Maximizer can play a vertex in $B$ as his first move. Thus if $S$ denotes the set of all vertices played when the game ends (in either game), then the set $S$ is a maximal enclaveless set in $G$. By our earlier observations, such a set $S$ contains a vertex of $B$ and is therefore different from the set $A$. Since the set $A$ is the unique $\psi$-set of $G$, this implies that $|S|>\psi(G)$. Therefore,

$$
|S| \geq \psi(G)+1=(n-\Gamma(G))+1=n-\frac{1}{2}(n+1)+1=\frac{1}{2}(n+1) .
$$

Since the first move of Maximizer from the set $B$ may not be an optimal move, we have that $\Psi_{g}^{+}(G) \geq \psi(G)+1$ if we are playing the Maximizer-start enclaveless game and $\Psi_{g}^{-}(G) \geq \psi(G)+1$ if we are playing the Minimizer-start enclaveless game. Thus, in both games Maximizer has a strategy to finish the game in at least $\frac{1}{2}(n+1)$ moves. Hence, if we assume that $\operatorname{IR}(G)=\frac{1}{2}(n+1)$, then $\Psi_{g}^{+}(G) \geq \min \left\{\Psi_{g}^{+}(G), \Psi_{g}^{-}(G)\right\} \geq \frac{1}{2}(n+1)$.

By Theorem 5.8(a), we note that Conjecture 1.2 holds for connected claw-free graphs. Moreover by Theorem 5.8(b), we note that Conjecture 1.3 holds for connected claw-free graphs even if we relax the minimum degree two condition and replace it with the requirement that the graph is isolate-free and different from the path $P_{3}$.

\section{Open problems and conjectures}

In this paper, we have shown that the $\frac{1}{2}$-Enclaveless Game Conjecture (see, Conjecture 1.2) is true for special classes of graphs, such as regular graphs and claw-free graphs. However, the conjecture has yet to be solved in general. It would be very interesting to prove or disprove the conjecture, or at least prove the conjecture for certain other important classes of graphs. We have also shown that the related conjecture for the Minimizer-start enclaveless game (see, Conjecture 1.3) holds for special classes. Again, it would be interesting to make 
further inroads into the conjecture. We close with the following two questions that we have yet to settle.

Question 6.1. Do there exist graphs $G$ such that $\Psi_{g}^{-}(G)>\Psi_{g}^{+}(G)$ ? If so, how large can the difference $\Psi_{g}^{-}(G)-\Psi_{g}^{+}(G)$ be made? Or is $\Psi_{g}^{-}(G) \leq \Psi_{g}^{+}(G)$ always true?

Question 6.2. Is it possible to characterize graphs $G$ such that $\Psi_{g}^{-}(G)=\Psi_{g}^{+}(G)$ ?

\section{ORCID iDs}

Michael A. Henning (D) https://orcid.org/0000-0001-8185-067X

Douglas F. Rall (D) https://orcid.org/0000-0002-5482-756X

\section{References}

[1] B. Brešar, M. A. Henning, S. Klavžar and D. F. Rall, Domination Games Played on Graphs, SpringerBriefs in Mathematics, Springer, 2021, doi:10.1007/978-3-030-69087-8.

[2] B. Brešar, S. Klavžar and D. F. Rall, Domination game and an imagination strategy, SIAM J. Discrete Math. 24 (2010), 979-991, doi:10.1137/100786800.

[3] C. Bujtás, Domination game on forests, Discrete Math. 338 (2015), 2220-2228, doi:10.1016/j. disc.2015.05.022.

[4] C. Bujtás, On the game domination number of graphs with given minimum degree, Electron. J. Combin. 22 (2015), \#P3.29 (18 pages), doi:10.37236/4497.

[5] E. J. Cockayne, S. T. Hedetniemi and D. J. Miller, Properties of hereditary hypergraphs and middle graphs, Canad. Math. Bull. 21 (1978), 461-468, doi:10.4153/cmb-1978-079-5.

[6] O. Favaron, Independence and upper irredundance in claw-free graphs, Discrete Appl. Math. 132 (2003), 85-95, doi:10.1016/s0166-218x(03)00392-5.

[7] A. Finbow, B. Hartnell and R. Nowakowski, Well-dominated graphs: a collection of wellcovered ones, Ars Combin. 25 (1988), 5-10.

[8] W. Goddard and M. A. Henning, The competition-independence game in trees, J. Combin. Math. Combin. Comput. 104 (2018), 161-170.

[9] M. A. Henning, My favorite domination game conjectures, in: R. Gera, T. W. Haynes and S. T. Hedetniemi (eds.), Graph Theory: Favorite Conjectures and Open Problems - 2, Springer, Cham, Problem Books in Mathematics, pp. 135-148, 2018, doi:10.1007/978-3-319-97686-0_ 12 .

[10] M. A. Henning and W. B. Kinnersley, Domination game: a proof of the $3 / 5$-conjecture for graphs with minimum degree at least two, SIAM J. Discrete Math. 30 (2016), 20-35, doi: $10.1137 / 140976935$.

[11] M. A. Henning and C. Löwenstein, Domination game: extremal families for the $3 / 5$-conjecture for forests, Discuss. Math. Graph Theory 37 (2017), 369-381, doi:10.7151/dmgt.1931.

[12] W. B. Kinnersley, D. B. West and R. Zamani, Extremal problems for game domination number, SIAM J. Discrete Math. 27 (2013), 2090-2107, doi:10.1137/120884742.

[13] G. Košmrlj, Realizations of the game domination number, J. Comb. Optim. 28 (2014), 447-461, doi:10.1007/s10878-012-9572-x.

[14] G. Košmrlj, Domination game on paths and cycles, Ars Math. Contemp. 13 (2017), 125-136, doi:10.26493/1855-3974.891.e93. 
[15] J. B. Phillips and P. J. Slater, An introduction to graph competition independence and enclaveless parameters, Graph Theory Notes N. Y. 41 (2001), 37-41.

[16] J. B. Phillips and P. J. Slater, Graph competition independence and enclaveless parameters, in: Proceedings of the Thirty-third Southeastern International Conference on Combinatorics, Graph Theory and Computing (Boca Raton, FL, 2002), volume 154, 2002 pp. 79-100.

[17] S. Schmidt, The $3 / 5$-conjecture for weakly $S\left(K_{1,3}\right)$-free forests, Discrete Math. 339 (2016), 2767-2774, doi:10.1016/j.disc.2016.05.017.

[18] S. J. Seo and P. J. Slater, Competition parameters of a graph, AKCE Int. J. Graphs Comb. 4 (2007), 183-190.

[19] P. J. Slater, Enclaveless sets and MK-systems, J. Res. Nat. Bur. Standards 82 (1977), 197-202, doi:10.6028/jres.082.019.

[20] J. Southey and M. A. Henning, Edge weighting functions on dominating sets, J. Graph Theory 72 (2013), 346-360, doi:10.1002/jgt.21649. 